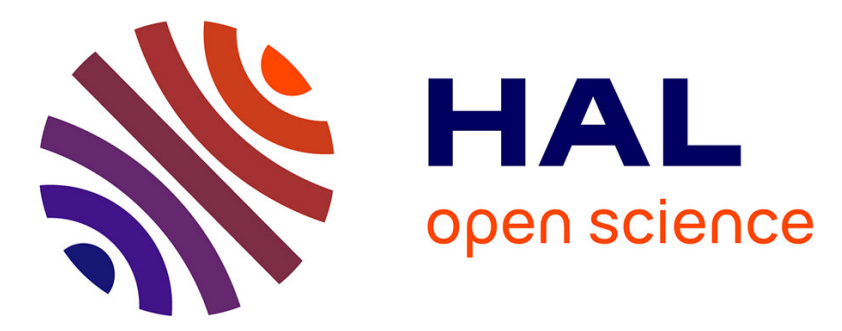

\title{
Comparaison de l'échographique des enthèses dans la polyarthrite rhumatoïde et la spondyloarthrite
}

Esther Ebstein, Baptiste Coustet, Vanina Masson-Behar, Marine Forien, Elisabeth Palazzo, Philippe Dieudé, Sébastien Ottaviani

\section{To cite this version:}

Esther Ebstein, Baptiste Coustet, Vanina Masson-Behar, Marine Forien, Elisabeth Palazzo, et al.. Comparaison de l'échographique des enthèses dans la polyarthrite rhumatoïde et la spondyloarthrite. Revue du Rhumatisme, 2019, 86, pp.269 - 274. 10.1016/j.rhum.2019.02.006 . hal-03484581

\section{HAL Id: hal-03484581 \\ https://hal.science/hal-03484581}

Submitted on 20 Dec 2021

HAL is a multi-disciplinary open access archive for the deposit and dissemination of scientific research documents, whether they are published or not. The documents may come from teaching and research institutions in France or abroad, or from public or private research centers.
L'archive ouverte pluridisciplinaire HAL, est destinée au dépôt et à la diffusion de documents scientifiques de niveau recherche, publiés ou non, émanant des établissements d'enseignement et de recherche français ou étrangers, des laboratoires publics ou privés.

\section{(ㅇ)(1) $\$$}

Distributed under a Creative Commons Attribution - NonCommercial| 4.0 International 


\title{
Échographique des enthèses dans la polyarthrite rhumatoïde et la spondyloarthrite
}

Esther Ebstein $^{\mathrm{a}, \mathrm{b}}$, Baptiste Coustet ${ }^{\mathrm{a}, \mathrm{b}}$, Vanina Masson-Behar ${ }^{\mathrm{a}, \mathrm{b}}$, Marine Forien ${ }^{\mathrm{a}, \mathrm{b}}$, Elisabeth Palazzo $^{\mathrm{a}, \mathrm{b}}$, Philippe Dieudéa,b et Sébastien Ottaviani ${ }^{\mathrm{a}, \mathrm{b}}$

\author{
${ }^{a}$ Université Paris Diderot, Sorbonne Paris Cité, UFR de Médecine, 75025 Paris, France \\ ${ }^{\mathrm{b}}$ Service de rhumatologie, hôpital Bichat, AP-HP, 75018 Paris, France
}

Correspondance : Sébastien Ottaviani, service de rhumatologie, hôpital Bichat, 46 rue Henri Huchard 75018 Paris, France.

E-mail : sebastien.ottaviani@aphp.fr

Téléphone : +33140258330 


\section{Résumé}

Objectif: nous avons souhaité comparer la prévalence de l'enthésopathie observée à l'échographie chez des patients atteints de spondyloarthrite (SpA) et de polyarthrite rhumatoïde (PR) et chez des témoins sains (TS).

Méthodes : une évaluation clinique et échographique des enthèses a été réalisée sur sept sites (tendon quadricipital, tendon patellaire proximal et distal, tendon d'Achille, aponévrose plantaire, tendon du triceps et épicondyliens latéraux) chez tous les patients atteints de PR (critères 2010 de l'ACR/EULAR) et de SpA (critères de l'ASAS) ainsi que chez les témoins. Les scores d'évaluation échographique des enthèses de Glasgow (GUESS) et de Madrid (MASEI) ont été déterminés par deux échographistes en aveugle des données cliniques.

Résultats : nous avons inclus 30 patients atteints de PR (âge moyen 55,7 $\pm 14,8$ ans, durée moyenne de la maladie 10,5 $\pm 7,9$ ans), 41 patients atteints de SpA (âge moyen 45,3 $\pm 15,4$ ans, durée moyenne de la maladie 9,2 $\pm 8,7$ ans) et 26 témoins (âge moyen $50,4 \pm 17,3$ ans). La prévalence des enthèses douloureuses sur les sites examinés était similaire dans les groupes SpA et PR (17\% contre $14 \%$, non significatif $[n s])$ mais supérieure à celle observée chez les témoins $(3 \%, p<0,05$ pour la comparaison avec la PR et la SpA). La comparaison des patients atteints de SpA et de PR a montré au moins une anomalie échographique des enthèses constatée avec une fréquence analogue (46\% et $48 \%$ des sites $[n s])$ mais la fréquence des anomalies à l'échographie était plus élevée dans les deux maladies rhumatismales que chez les TS $(31 \%, p<0,05$ pour la comparaison avec la PR et la SpA). Le score MASEI moyen était de 8,5 \pm 7,3 dans la PR, de 7,8 \pm 6,5 dans la SpA (ns) et de 3,4 $\pm 2,8$ chez les TS ( $p<0,05$ pour la comparaison avec la PR et la SpA). Au total, six patients du groupe PR $(20 \%)$ et quatre du groupe SpA (10\%) avaient un score MASEI $\geq 18$ (ns). Aucun des TS n'avait un score MASEI $\geq 18(p<0,05$ pour la comparaison avec la PR et 
la SpA). Le score GUESS moyen était de 5,8 \pm 3,1 dans la PR, de 6,3 \pm 3,9 dans la SpA (ns) et de 4,0 $\pm 3,1$ chez les TS ( $p<0,01$ pour la comparaison avec la SpA et $p<0,05$ pour la comparaison avec la PR).

Conclusion: il n'y avait pas de différences entre les patients atteints de PR et de SpA concernant les anomalies des enthèses visibles à l'échographie. La spécificité de ces caractéristiques échographiques peut être faible dans les affections inflammatoires touchant les articulations et les enthèses, comme la SpA ou la PR.

Mots clés : échographie, enthésopathie, enthésite, spondyloarthrite, polyarthrite rhumatoïde. 


\section{Introduction}

L'enthésopathie se caractérise par une inflammation des insertions osseuses des tendons, ligaments ou capsules. C'est une manifestation fréquente dans la spondyloarthrite (SpA) qui pourrait être recherchée pour la classification diagnostique de la SpA [1] et la prise en charge thérapeutique [2]. Il peut toutefois être difficile de reconnaître une enthésopathie en raison de la faiblesse de la sensibilité et de la spécificité de l'examen clinique. L'European League Against Rheumatism (EULAR) recommande d'utiliser l'imagerie par résonance magnétique (IRM) ou l'échographie pour détecter l'enthésopathie.

Le recours à l'IRM étant limité par sa disponibilité restreinte, l'échographie paraît une méthode intéressante pour la détection de l'enthésopathie. L'échographie en mode B est un outil performant pour identifier des changements structuraux au niveau des enthèses, notamment érosions, bursite, calcification, épaississement ou hypo-échogénicité [3]. Le mode Doppler Puissance (DP) permet de visualiser l'hypervascularisation. Selon certaines études, l'évaluation échographique de l'enthésopathie pourrait être une méthode sensible et spécifique pour le diagnostic de la SpA [4-7]. Toutefois, la performance diagnostique de cette technique semble variable et d'autres études ont décrit une prévalence similaire de l'enthésopathie échographique dans la PR et la SpA [8] ou le rhumatisme psoriasique (RPso) [9]. De plus, l'évaluation échographique des talons n'a pas permis de différencier les patients atteints de SpA des témoins [10]. Cette variabilité des résultats peut suggérer un manque de spécificité de l'échographie pour détecter des caractéristiques d'enthésopathie ou un manque de spécificité des scores d'évaluation.

Plusieurs scores semi-quantitatifs ont été mis au point pour quantifier l'enthésopathie. Les études utilisent le plus souvent l'index d'évaluation échographique de Madrid (MASEI) [6] et celui de Glasgow (GUESS) [10] ; par ailleurs, les principaux sites d'enthèse étudiés 
sont le tendon du triceps, les épicondyliens latéraux et les insertions tendineuses des membres inférieurs.

Nous avons souhaité déterminer la prévalence de l'enthésopathie détectée par échographie chez des patients atteints de $\mathrm{SpA}$ et de PR par comparaison avec des témoins sains afin d'estimer l'utilité des principaux scores d'évaluation échographique des enthèses chez des patients présentant une maladie inflammatoire.

\section{Méthodes}

\subsection{Patients et conception de l'étude}

Nous avons mené une étude transversale monocentrique incluant des patients atteints de PR selon les critères 2010 de l'ACR/EULAR [11], des patients atteints de SpA selon les critères de l'ASAS [1] et des témoins sains ne présentant pas d'affection rhumatismale (TS). Tous les patients ont été recrutés consécutivement sur une période de six mois dans le service de rhumatologie de l'hôpital Bichat (Paris). Les données suivantes ont été collectées : sexe, âge, durée de la maladie, nombre et sites des enthèses douloureuses, intensité de la douleur sur une échelle visuelle analogique (EVA, 0-100 mm), prise d'un traitement de fond antirhumatismal (DMARD), de corticoïdes ou d'un traitement biologique antérieur, vitesse de sédimentation (VS) et taux de protéine réactive C (CRP). Pour les patients atteints de PR, les données suivantes ont également été collectées : nombre d'articulations douloureuses (NAD) et gonflées (NAG) sur 28 sites, positivité des anticorps antipeptides cycliques citrullinés (ACPA) et du facteur rhumatoïde (FR), présence d'érosions et score d'activité de la maladie (DAS 28). Pour les patients atteints de SpA, nous avons également répertorié la positivité de 
l'allèle HLA-B27, l'indice d'activité BASDAI (Bath Ankylosing Spondylitis Disease Activity Index) et l'indice fonctionnel BASFI (Bath Ankylosing Spondylitis Functional Index).

\subsection{Déclaration éthique}

L'étude a été approuvée par le comité d'examen institutionnel local ( $\left.\mathrm{n}^{\mathrm{o}} 12-011\right)$ et le consentement éclairé écrit de tous les participants a été obtenu.

\section{3. Évaluation clinique}

L'insertion distale du tendon du quadriceps fémoral (TQF), les insertions proximale (IPTP) et distale (IDTP) du tendon patellaire, l'aponévrose plantaire (AP), les tendons d'Achille (TA), l'insertion distale du tendon du triceps et les épicondyliens latéraux (EL) ont été évalués sur les deux membres. La douleur a été évaluée en exerçant une pression localisée. L'examen clinique a été réalisé par un rhumatologue en aveugle de l'évaluation échographique.

\section{4. Évaluation échographique}

L'évaluation échographique a été standardisée et effectuée le même jour que l'évaluation clinique par deux rhumatologues formés ( $\mathrm{SO}$ et $\mathrm{BC}$ ) au moyen d'un échographe Esaote MyLab70 (Gênes, Italie) à sondes linéaires 5-12 MHz (genoux) et 12-18 MHz (pieds et coudes). L'évaluation échographique a été réalisée le matin, après 30 minutes de repos. La température de la pièce a été maintenue à $20^{\circ} \mathrm{C}$. L'évaluateur échographique n'avait pas connaissance des données cliniques. L'épaisseur du tendon, son échogénicité et la présence de calcifications, d'enthésophytes, de bursite et d'érosions ont été évaluées en mode B (figure 1). Pour évaluer la vascularisation des enthèses, le mode DP a été utilisé avec une fréquence de répétition des impulsions de $750 \mathrm{~Hz}$ et le gain a été ajusté de manière à éliminer le bruit de fond. Chaque site d'enthèse a été évalué dans deux plans : longitudinal et transverse. 
Conformément aux critères des scores de MASEI [6] et du GUESS [10], nous avons procédé à l'analyse bilatérale des structures suivantes : patella (aux insertions des tendons du quadriceps fémoral et patellaire), insertions du tendon d'Achille et de l'aponévrose plantaire sur le calcanéus et insertion du tendon du triceps sur le processus olécrânien. Nous avons également effectué une évaluation échographique des enthèses des épicondyliens latéraux selon des descriptions antérieures [4,12]. Chaque examen a duré environ 20 minutes. Les patients ont été placés en décubitus dorsal, genou fléchi à $30^{\circ}$, pour évaluer les enthèses patellaires et du quadriceps.

Les tendons d'Achille et l'aponévrose plantaire ont été examinés en décubitus ventral, pieds fléchis à $90^{\circ}$ dépassant du bord de la table ou en décubitus dorsal avec les genoux et les chevilles fléchis à $90^{\circ}$. Pour l'évaluation des épicondyliens latéraux, la main était posée en pronation sur la table, le coude fléchi à $90^{\circ}$. Pour l'examen de l'insertion tricipitale, le coude était fléchi à $90^{\circ}$.

L'échographie a évalué l'épaisseur des tendons et leur échogénicité, les érosions osseuses, la présence d'une bursite, la vascularisation en mode DP et la présence de calcifications et d'enthésophytes. La bursite a été définie comme une zone anéchogène ou hypo-échogène localisée et bien délimitée sur le site d'une bourse anatomique, compressible par la sonde. L'épaisseur de l'enthèse a été mesurée à l'insertion du bord du tendon le plus profond dans l'os selon un axe longitudinal. Conformément aux préconisations du score MASEI, le signal DP a été évalué au niveau de la bourse ou de l'enthèse (au niveau de l'insertion corticale et en intra-tendineux).

Les scores GUESS et MASEI ont été calculés comme décrit précédemment $[6,10]$.

\subsection{Analyse statistique}


Les variables continues sont exprimées en moyenne (ET) ou médiane (intervalle interquartile [IQR]). Les variables catégorielles sont exprimées en fréquence (\%). La comparaison des variables catégorielles a été réalisée au moyen du test $\chi^{2}$ de Pearson ou du test de Fisher et celle des variables continues, par le test $t$ de Student ou le test de la somme des rangs de Wilcoxon. La fiabilité intra et interobservateur de l'imagerie a été calculée sur les images de dix patients. Les données ont été analysées une nouvelle fois en conditions d'aveugle au moins un mois après la première évaluation. L'accord intra et interobservateur pour l'échographie a été estimé par le coefficient $\kappa$, avec le barème suivant : > 0,8, presque parfait ; de 0,6 à 0,8 , fort ; de 0,4 à 0,6 , modéré ; de 0,2 à 0,4 , faible $; \leq 0,2$, très faible ; <0, désaccord.

\subsection{Financement}

L'achat de l'échographe a été financé par Roche-Chugai.

\section{Résultats}

\subsection{Caractéristiques des patients}

Les caractéristiques cliniques de la population totale sont présentées dans le tableau 1 . Au total 30 patients atteints de PR (17\% d'hommes, âge moyen 55,7 $\pm 14,8$ ans, $93 \%$ ACPA+), 41 patients atteints de SpA (68\% d'hommes, âge moyen 45,3 \pm 15,4 ans, $64 \% H L A-B 27+$ ) et 26 TS (34 \% d'hommes, âge moyen 50,4 \pm 17,3 ans) ont été inclus de façon consécutive.

\subsection{Examen clinique des enthèses}

L'examen clinique a révélé des enthèses douloureuses sur 98 des 574 sites examinés (17\%) dans le groupe $\mathrm{SpA}, 59 / 420$ sites $(14 \%)$ dans le groupe PR (non significatif $[n s]$ ) et 
11/364 sites $(3 \%)$ chez les témoins ( $p<0,05$ pour la comparaison avec la PR et la SpA) (tableau 2). Dans les groupes SpA et PR, les patients avaient en moyenne respectivement 2,4 et 1,96 sites d'enthèse douloureux $(n s)$, contre 0,44 dans le groupe témoin $(p<0,05$ pour la comparaison avec la PR et la SpA). Le nombre médian de sites douloureux par patient était de 2 pour la SpA, 1 pour la PR et 0 chez les TS.

\section{3. Évaluation échographique des enthèses (tableau 3)}

Le score MASEI moyen était de 8,5 \pm 7,3 dans la PR, de 7,8 $\pm 6,5$ dans la SpA $(n s)$ et de 3,4 $\pm 2,8$ chez les TS ( $p<0,05$ pour la comparaison avec la PR et la SpA) (figure 2). Six patients du groupe PR $(20 \%)$ et quatre du groupe SpA (10\%) (ns) avaient un score MASEI $\geq 18$. Aucun des TS n'avait un score MASEI $\geq 18(p<0,05$ pour la comparaison avec la PR et la SpA). Le score GUESS moyen était de 5,8 $\pm 3,1$ dans la PR, de 6,3 \pm 3,9 dans la SpA ( $p$ $=0,67)$ et de 3,9 $\pm 3,0$ chez les TS ( $p<0,01$ pour la comparaison avec la SpA et $p<0,05$ pour la comparaison avec la PR). L'échographie a montré au moins une anomalie d'enthèse sur 264/574 sites (46\%) dans le groupe SpA, 200/420 sites (48\%) dans le groupe PR (ns) et 115/364 sites $(31 \%$ ) chez les témoins ( $p<0,05$ pour la comparaison avec la PR et la SpA). L'analyse individuelle de chaque caractéristique échographique de l'enthésopathie a montré une fréquence plus élevée dans la SpA que dans la PR pour l'épaisseur du tendon du triceps $(12,2 \%$ contre $1,7 \%, p<0,05)$, les EL $(13,4 \%$ contre $0 \%, p<0,01)$ et la positivité DP du TQF $(8,5 \%$ contre $0 \%, p<0,05)$. En revanche, les érosions osseuses en regard des EL étaient plus fréquentes chez les patients atteints de PR $(28,3 \%$ contre $2,4 \%, p<0,001)$.

L'analyse individuelle de chaque caractéristique échographique de l'enthésopathie a montré une fréquence plus élevée chez les patients atteints de SpA que chez les témoins pour l'épaisseur du tendon du triceps $(12,2 \%$ contre $1,9 \%, p<0,05)$, l'IDTP (93\% contre 67,3\%, $p<0,001)$ et le TQF $(19,5 \%$ contre $5,7 \%, p<0,05)$, ainsi que pour la positivité DP du TQF 
$(8,5 \%$ contre $0 \%, p<0,05)$, du TA $(8,5 \%$ contre $0 \%, p<0,05)$ et des EL $(28,1 \%$ contre $9,6 \%, p<0,05)$. Les enthésophytes étaient plus fréquents chez les patients atteints de SpA que chez les TS sur plusieurs sites : IPTP $(9,7 \%$ contre $0 \%, p<0,05)$, TA $(29,2 \%$ contre $9,6 \%, p<0,01)$, AP $(18,3 \%$ contre $5,7 \%, p<0,05)$ et triceps $(15,8 \%$ contre $3,8 \%, p$ $<0,05)$. Il n'y avait pas de différence statistique quant à l'hypo-échogénicité, les calcifications et les érosions.

La fiabilité intra et interobservateur était modérée (coefficient $\kappa: 0,51$ [intervalle de confiance à $95 \% 0,42-0,60]$ et $0,55[0,46-0,64])$.

\section{Discussion}

L'enthésopathie est une manifestation courante dans la SpA qui pourrait être recherchée pour les besoins de la classification diagnostique de cette pathologie [1], mais on la retrouve également dans la PR [8]. Dans cette étude, nous avons utilisé l'échographie pour analyser la prévalence des anomalies des enthèses dans ces deux affections inflammatoires chroniques. L'examen clinique a montré une prévalence relativement importante de douleurs des enthèses dans les deux maladies; la prévalence de l'enthésopathie observée par échographie était similaire pour les deux. Conformément à nos attentes, les anomalies des enthèses observées à l'échographie étaient plus nombreuses dans les deux maladies rhumatismales que chez les TS.

Plusieurs études ont démontré la pertinence de l'échographie pour évaluer l'enthésopathie chez les patients atteints de SpA [10,13]. L'échographie en échelle mode B peut détecter les changements structuraux caractéristiques de l'enthésopathie, notamment érosions, bursite, calcifications, épaississement ou hypo-échogénicité [3]. Le mode DP permet de visualiser une hypervascularisation. Selon certaines études, l'évaluation échographique de l'enthésopathie pourrait être une méthode sensible et spécifique pour le diagnostic de la SpA [4-7]. La 
définition de l'enthésopathie reste cependant mal établie [3]. Les anomalies ou les sites d'enthèses qu'il convient d'analyser restent à déterminer. Par ailleurs, les scores d'évaluation échographique des enthèses sont très nombreux. Les plus utilisés sont les scores MASEI et GUESS $[6,10]$. Une comparaison avec des patients témoins a montré qu'un score MASEI $>18$ était fortement évocateur de SpA [6]. Dans notre étude, ces deux scores n'ont pas permis de discriminer la PR et la SpA et la proportion de patients ayant un score MASEI > 18 était faible. Il est possible que ces deux scores ne soient pas sensibles pour l'enthésopathie et/ou que les anomalies des enthèses détectées à l'échographie ne soient pas spécifiques. Nos résultats concordent avec ceux précédemment publiés rapportant une faible prévalence de l'enthésopathie échographique dans les talons de patients atteints de SpA [14], et une similitude entre SpA et PR quant à l'enthésopathie détectée par l'échographie [8].

L'absence de discrimination de l'enthésopathie échographique entre PR et SpA peut s'expliquer par le fait que la PR s'accompagne parfois d'une atteinte des gaines synoviales et des tendons pouvant entraîner une réaction des enthèses [15]. Une autre étude comparant PR et rhumatisme psoriasique (RPso) n'a pas trouvé de différences en ce qui concerne l'atteinte des enthèses du calcanéus à l'échographie [16]. Les caractéristiques échographiques de l'enthésopathie peuvent être non spécifiques. Dans une précédente étude, la prévalence des enthésophytes du calcanéus à l'échographie était similaire pour l'arthrose, la PR et le RPso. Feydy et al. n'ont pas observé de différences pour l'enthésopathie échographique dans les talons entre des patients atteints de SpA et des témoins [14]. Qui plus est, le score échographique de l'enthésopathie peut augmenter de façon significative après 30 minutes de marche sur un tapis roulant chez des personnes en bonne santé [16].

Notre étude présente certaines limites. La première est le nombre relativement faible de patients. Néanmoins, nous avons évalué les membres supérieurs et inférieurs et calculé les deux scores MASEI et GUESS, pour un total de 937 sites. La fiabilité intra et interobservateur 
était modérée mais similaire à celle observée par Terslev et al., explorant la fiabilité de l'échographie pour l'évaluation de l'enthésite chez des patients atteints de SpA pour le compte de l'OMERACT [17]. Le score MASEI que nous avons obtenu était inférieur à celui rapporté par de précédentes études, peut-être parce que nous n'avons pas codé les enthésophytes comme des calcifications [18], ces calcifications ajoutant deux points par articulation (jusqu'à 10 points par patient) dans le MASEI. Nous avons considéré que les enthésophytes sont couramment décrits comme non liés à la présence d'une maladie inflammatoire [19]. Cette faible prévalence de l'enthésopathie échographique dans les deux populations pourrait aussi s'expliquer par la proportion élevée de patients recevant un traitement biologique, conduisant à sous-estimer l'enthésopathie. Toutefois, les patients atteints de PR étaient plus nombreux à recevoir un traitement biologique que ceux atteints de SpA, ce qui n'explique pas la prévalence comparable de l'enthésopathie échographique. Une autre limite de l'étude était l'absence de quantification de l'activité physique pouvant avoir une influence sur l'évaluation échographique des enthèses [20]. Pour limiter cet impact, l'évaluation échographique a été réalisée le matin, après une période de repos de 30 minutes. Enfin, nous n'avions pas d'information relative au statut $H L A-B 27$ pour les patients atteints de PR, mais Mera-Valera et al. n'ont observé aucune différence entre $H L A-B 27$ positif et négatif dans la PR [21].

En conclusion, nous n'avons pas constaté de différences entre les patients atteints de SpA et de PR quant aux anomalies des enthèses observées à l'échographie. La spécificité des caractéristiques échographiques des enthèses peut être faible dans les affections inflammatoires touchant les articulations et les enthèses, comme la SpA ou la PR. Des études supplémentaires sont nécessaires pour valider nos résultats.

\section{Remerciements}


Nous remercions Laura Smales (BioMedEditing) pour son travail éditorial.

Déclaration d'intérêts : les auteurs déclarent n'avoir aucun conflit d'intérêts. 


\section{Références}

[1] Rudwaleit M, Landewe R, van der Heijde D, et al.: The development of Assessment of SpondyloArthritis international Society classification criteria for axial spondyloarthritis (part I): classification of paper patients by expert opinion including uncertainty appraisal. Ann Rheum Dis 2009, 68:770-6.

[2] Braun J, van den Berg R, Baraliakos X, et al.: 2010 update of the ASAS/EULAR recommendations for the management of ankylosing spondylitis. Ann Rheum Dis 2011, 70:896-904.

[3] Gandjbakhch F, Conaghan PG, Ejbjerg B, et al.: Synovitis and osteitis are very frequent in rheumatoid arthritis clinical remission: results from an MRI study of 294 patients in clinical remission or low disease activity state. J Rheumatol 2011, 38:2039-44.

[4] D'Agostino MA, Aegerter P, Bechara K, et al.: How to diagnose spondyloarthritis early? Accuracy of peripheral enthesitis detection by power Doppler ultrasonography. Ann Rheum Dis 2011, 70:1433-40.

[5] D'Agostino MA, Said-Nahal R, Hacquard-Bouder C, et al.: Assessment of peripheral enthesitis in the spondylarthropathies by ultrasonography combined with power Doppler: a cross-sectional study. Arthritis Rheum 2003, 48:523-33.

[6] de Miguel E, Cobo T, Munoz-Fernandez S, et al.: Validity of enthesis ultrasound assessment in spondyloarthropathy. Ann Rheum Dis 2009, 68:169-74.

[7] de Miguel E, Munoz-Fernandez S, Castillo C, et al.: Diagnostic accuracy of enthesis ultrasound in the diagnosis of early spondyloarthritis. Ann Rheum Dis 2011, 70:434-9.

[8] Genc H, Cakit BD, Tuncbilek I, et al.: Ultrasonographic evaluation of tendons and enthesal sites in rheumatoid arthritis: comparison with ankylosing spondylitis and healthy subjects. Clin Rheumatol 2005, 24:272-7.

[9] Ibrahim G, Groves C, Chandramohan M, et al.: Clinical and ultrasound examination of the leeds enthesitis index in psoriatic arthritis and rheumatoid arthritis. ISRN Rheumatol 2011, 2011:731917.

[10] Balint PV, Kane D, Wilson H, et al.: Ultrasonography of entheseal insertions in the lower limb in spondyloarthropathy. Ann Rheum Dis 2002, 61:905-10.

[11] Aletaha D, Neogi T, Silman AJ, et al.: 2010 Rheumatoid arthritis classification criteria: an American College of Rheumatology/European League Against Rheumatism collaborative initiative. Arthritis Rheum 2010, 62:2569-81.

[12] Gandjbakhch F, Terslev L, Joshua F, et al.: Ultrasound in the evaluation of enthesitis: status and perspectives. Arthritis Res Ther 2011, 13:R188.

[13] Lehtinen A, Taavitsainen $\mathrm{M}$ and Leirisalo-Repo $\mathrm{M}$ : Sonographic analysis of enthesopathy in the lower extremities of patients with spondylarthropathy. Clin Exp Rheumatol 1994, 12:143-8.

[14] Feydy A, Lavie-Brion MC, Gossec L, et al.: Comparative study of MRI and power Doppler ultrasonography of the heel in patients with spondyloarthritis with and without heel pain and in controls. Ann Rheum Dis 2012, 71:498-503. 
[15] Scutellari PN and Orzincolo C: Rheumatoid arthritis: sequences. Eur J Radiol 1998, 27 Suppl 1:S31-8.

[16] Meric JC, Grandgeorge Y, Lotito G, et al.: Walking before an ultrasound assessment increases the enthesis score significantly. J Rheumatol 2011, 38:961.

[17] Terslev L, Naredo E, Iagnocco A, et al.: Defining enthesitis in spondyloarthritis by ultrasound: results of a Delphi process and of a reliability reading exercise. Arthritis Care Res (Hoboken) 2014, 66:741-8.

[18] Eder L, Jayakar J, Thavaneswaran A, et al.: Is the MAdrid Sonographic Enthesitis Index useful for differentiating psoriatic arthritis from psoriasis alone and healthy controls? J Rheumatol 2014, 41:466-72.

[19] Shaibani A, Workman R and Rothschild BM: The significance of enthesopathy as a skeletal phenomenon. Clin Exp Rheumatol 1993, 11:399-403.

[20] Lanfranchi MA, Leluc O, Tavano A, et al.: Are Ultrasound Findings Similar in Patients with Axial Spondyloarthritis and in Athlete Entheses? J Rheumatol 2017, 44:609-612.

[21] Mera-Varela A, Ferreiro-Iglesias A, Perez-Pampin E, et al.: Ultrasonographic assessment of enthesitis in HLA-B27 positive patients with rheumatoid arthritis, a matched case-only study. PLoS One 2013, 8:e58616. 


\section{Légende des figures :}

Figure 1. Caractéristiques échographiques de l'enthésopathie. Vue longitudinale de l'enthèse $d u$ tendon du triceps montrant des érosions chez un patient atteint de PR (A) et un autre atteint de $\operatorname{SpA}(B)$. Érosion de l'enthèse du tendon d'Achille en vue longitudinale associée à une hypo-échogénicité et à la positivité Doppler Puissance chez un patient atteint de SpA (C) et érosion sur le même site chez un patient atteint de $P R(D)$. Épaississement associé à la positivité DP du tendon du quadriceps $(E)$ et DP de l'épicondylien latéral $(F)$ chez un patient atteint de SpA.

Figure 2. Scores GUESS et MASEI des trois populations

p représente le test de Wilcoxon.

SpA : spondyloarthrite; $P R$ : polyarthrite rhumatö̈de; TS: témoins sains; ns: nonsignificatif 


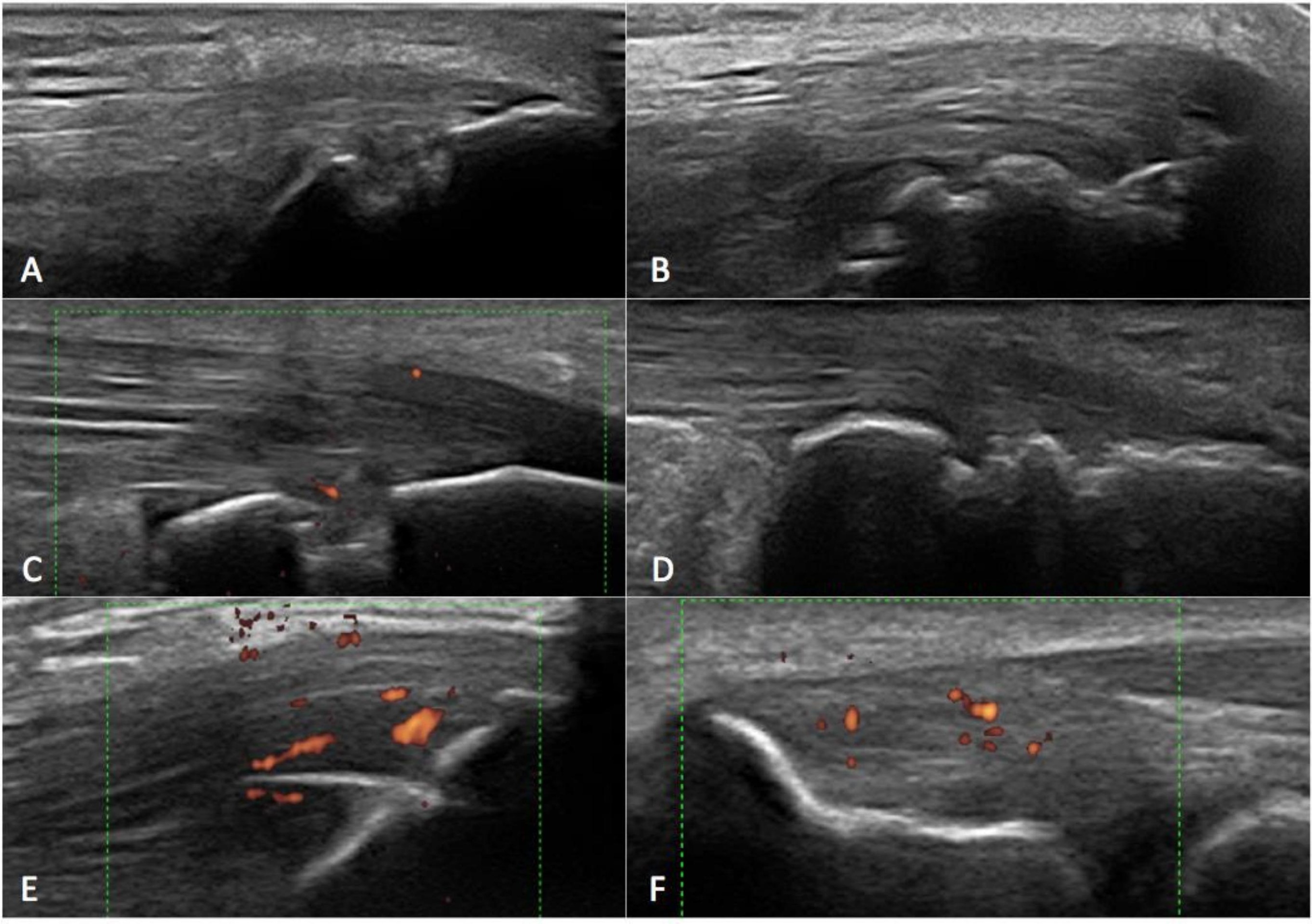




$$
P<0,01
$$

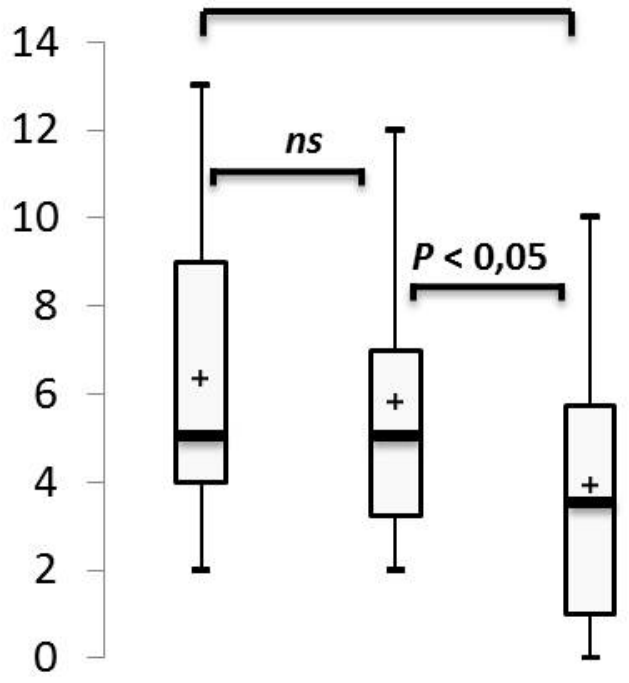

GUESS SpA GUESS RA GUESS TS

$$
P<0.05
$$

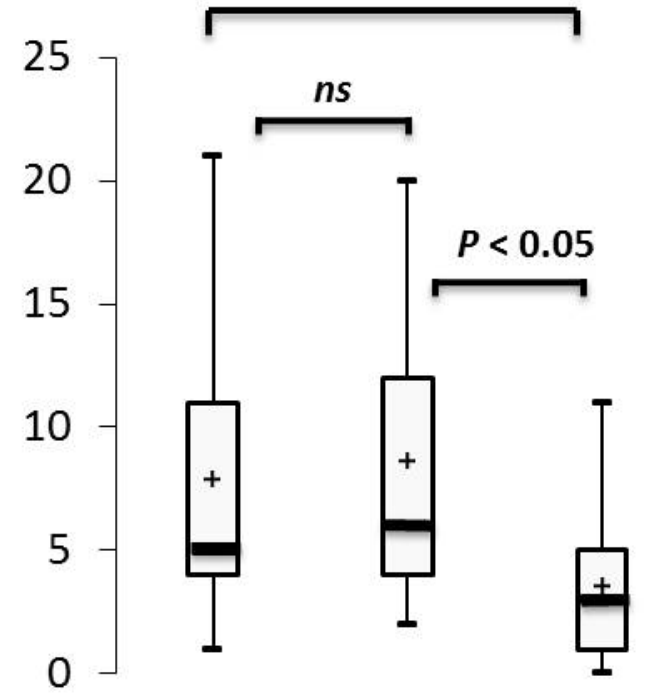

MASEI SPA MASEI RA MASEI TS 
Tableau 1. Caractéristiques des patients atteints de spondyloarthrite ( $\mathrm{SpA}$ ) et de polyarthrite rhumatoïde (PR) et des témoins en bonne santé (TS).

\begin{tabular}{|c|c|c|c|}
\hline & $\begin{array}{c}\text { SpA } \\
(\mathrm{n}=41)\end{array}$ & $\begin{array}{c}\text { PR } \\
(\mathrm{n}=30)\end{array}$ & $\begin{array}{c}\text { TS } \\
(\mathrm{n}=26)\end{array}$ \\
\hline Âge, ans & $45,3 \pm 15,4$ & $55,7 \pm 14,8$ & $50,4 \pm 17,3$ \\
\hline Sexe, n (\% d'hommes) & $28(68)$ & $5(17)$ & $9(34)$ \\
\hline Durée de la maladie, ans & $9,2 \pm 8,7$ & $10,5 \pm 7,9$ & - \\
\hline Positivité $H L A-B 27, \mathrm{n}(\%)$ & $28(67)$ & SO & SO \\
\hline Psoriasis, $\mathrm{n}(\%)$ & $10(24)$ & SO & $\mathrm{SO}$ \\
\hline Positivité ACPA, n (\%) & SO & $28(93)$ & SO \\
\hline $\begin{array}{l}\text { Positivité du facteur rhumatoïde, } \mathrm{n} \\
(\%)\end{array}$ & $\mathrm{SO}$ & $25(83)$ & $\mathrm{SO}$ \\
\hline $\mathrm{VS}, \mathrm{mm}$ & $24,7 \pm 26,7$ & $21,5 \pm 21,9$ & $\mathrm{SO}$ \\
\hline Taux de CRP, mg/l & $28,2 \pm 49,3$ & $15,6 \pm 29$ & $\mathrm{SO}$ \\
\hline BASDAI (/10) mm & $3,5 \pm 2,4$ & SO & SO \\
\hline BASFI (/10) mm & $2,9 \pm 2,5$ & SO & SO \\
\hline DAS 28 & SO & $3,4 \pm 1,9$ & $\mathrm{SO}$ \\
\hline $\begin{array}{l}\text { Traitement biologique, } \mathrm{n}(\%) \\
\text { Anti-TNF } \alpha \\
\text { Autres agents biologiques }\end{array}$ & $\begin{array}{c}18(43) \\
18(43) \\
0(0)\end{array}$ & $\begin{array}{c}17(56) \\
3(10) \\
14(46)\end{array}$ & 0 \\
\hline Prise de DMARD, n $(\%)$ & $10(24)$ & $25(83)$ & 0 \\
\hline Prise de corticoïdes, $\mathrm{n}(\%)$ & $1(2,5)$ & $19(63)$ & $\mathrm{SO}$ \\
\hline Prise d'AINS, n (\%) & $20(49)$ & SO & SO \\
\hline
\end{tabular}

Les données sont exprimées en moyenne \pm ET sauf indication contraire.

ACPA : anticorps antipeptides cycliques citrullinés ; VS : vitesse de sédimentation ; CRP : protéine réactive C ; BASDAI : indice d'activité Bath Ankylosing Spondylitis Disease Activity Index; BASFI : indice fonctionnel Bath Ankylosing Spondylitis Functional Index; DAS 28 : score d'activité de la maladie dans 28 articulations ; TNF : facteur de nécrose tumorale ; DMARD : traitement de fond antirhumatismal ; AINS : anti-inflammatoires non stéroïdiens ; SO : sans objet. 
Tableau 2. Sites d'enthèse douloureux chez les patients atteints de SpA et de PR et les témoins en bonne santé.

\begin{tabular}{lcccccc}
\hline & $\begin{array}{c}\text { SpA } \\
(\mathrm{n}=82)\end{array}$ & $\begin{array}{c}\mathrm{PR} \\
(\mathrm{n}=60)\end{array}$ & $\begin{array}{c}\text { Témoins } \\
(\mathrm{n}=52)\end{array}$ & $\begin{array}{c}\text { SpA } \\
\text { contre PR } \\
\mathrm{p}^{*}\end{array}$ & $\begin{array}{c}\text { SpA contre } \\
\text { témoins } \\
\mathrm{p}^{*}\end{array}$ & $\begin{array}{c}\text { PR contre } \\
\text { témoins } \\
\mathrm{p}^{*}\end{array}$ \\
\hline Épicondyles latéraux & $29(35)$ & $12(20)$ & $3(5,8)$ & $\mathrm{p}=0,06$ & $\mathbf{p}=\mathbf{5 , 1 0 - 5}$ & $\mathbf{p}=\mathbf{0 , 0 4}$ \\
$\begin{array}{l}\text { Insertion du tendon du } \\
\text { triceps }\end{array}$ & $12(14,6)$ & $6(10)$ & $4(7,7)$ & $\mathrm{p}=0,45$ & $\mathrm{p}=0,28$ & $\mathrm{p}=0,74$ \\
Quadriceps fémoral & $6(7,5)$ & $8(13,5)$ & $2(3,8)$ & $\mathrm{p}=0,26$ & $\mathrm{p}=0,48$ & $\mathrm{p}=0,1$ \\
$\begin{array}{l}\text { Tendon patellaire } \\
\text { proximal }\end{array}$ & $12(14,6)$ & $11(18)$ & $1(1,9)$ & $\mathrm{p}=0,64$ & $\mathbf{p}=\mathbf{0 , 0 1}$ & $\mathbf{p}=\mathbf{0 , 0 0 5}$ \\
$\begin{array}{l}\text { Tendon patellaire distal } \\
\text { Tendon d'Achille }\end{array}$ & $9(11)$ & $5(8,5)$ & $0(0)$ & $\mathrm{p}=0,77$ & $\mathbf{p}=\mathbf{0 , 0 1}$ & $\mathrm{p}=0,06$ \\
Fascia plantaire & $14(17)$ & $10(16,5)$ & $0(0)$ & $\mathrm{p}=1$ & $\mathbf{p}=\mathbf{0 , 0 0 0 8}$ & $\mathbf{p}=\mathbf{0 , 0 1}$ \\
\hline
\end{tabular}

Les données sont présentées en nombre (\%) de sites.

* Test exact de Fisher bilatéral. 
Tableau 3. Évaluation échographique de l'enthésopathie chez les patients atteints de SpA et de PR et les TS.

\begin{tabular}{|c|c|c|c|c|c|c|c|}
\hline & TQF & IPTP & IDTP & TA & FP & Triceps & EL \\
\hline \multicolumn{8}{|l|}{$\operatorname{SpA}(n=82)$} \\
\hline Épaisseur & $16(19,5)$ & $51(61,0)$ & $76(93,0)$ & $10(12,2)$ & $10(12,2)$ & $10(12,2)$ & $11(13,4)$ \\
\hline $\begin{array}{l}\text { Hypo- } \\
\text { échogénicité }\end{array}$ & $2(2,4)$ & $1(1,2)$ & $2(2,4)$ & $5(6,1)$ & $3(3,6)$ & $2(2,4)$ & $16(19,5)$ \\
\hline Positivité DP & $7(8,5)$ & $2(2,4)$ & $4(4,9)$ & $7(8,5)$ & $0(0)$ & $9(11,0)$ & $23(28,1)$ \\
\hline Calcifications & $1(1,2)$ & $0(0)$ & $4(4,89)$ & $1(1,2)$ & $1(1,2)$ & $1(1,2)$ & $3(3,6)$ \\
\hline Enthésophytes & $26(31,7)$ & $8(9,7)$ & $4(4,9)$ & $24(29,2)$ & $15(18,3)$ & $13(15,8)$ & $9(11,0)$ \\
\hline Érosions & $4(4,9)$ & $5(6,1)$ & $3(3,6)$ & $6(7,5)$ & $1(1,2)$ & $0(0)$ & $2(2,4)$ \\
\hline Score MASEI & 0,55 & 0,81 & 1,20 & 0,59 & 0,20 & 0,47 & SO \\
\hline Score GUESS & 0,57 & 0,73 & 1,05 & 0,50 & 0,3 & $\mathrm{SO}$ & $\mathrm{SO}$ \\
\hline \multicolumn{8}{|l|}{ PR $(n=60)$} \\
\hline Épaisseur & $7(11,7)$ & $40(66,7)$ & $54(90,0)$ & $6(10,0)$ & $8(13,3)$ & $1(1,7)$ & $0(0)$ \\
\hline $\begin{array}{l}\text { Hypo- } \\
\text { échogénicité }\end{array}$ & $1(1,7)$ & $1(1,7)$ & $4(6,7)$ & $4(6,7)$ & $1(1,7)$ & $3(5,0)$ & $9(15,0)$ \\
\hline Positivité DP & $0(0)$ & $5(8,3)$ & $6(10,0)$ & $5(8,3)$ & $0(0)$ & $8(13,3)$ & $16(26,7)$ \\
\hline Calcifications & $1(1,7)$ & $1(1,7)$ & $3(5,0)$ & $2(3,3)$ & $2(3,3)$ & $1(1,7)$ & $8(13,3)$ \\
\hline Enthésophytes & $12(20,0)$ & $3(5,0)$ & $3(5,0)$ & $18(30,0)$ & $1(1,67)$ & $13(21,7)$ & $6(10,0)$ \\
\hline Érosions & $0(0)$ & $2(3,3)$ & $0(0)$ & $5(8,3)$ & $2(3,3)$ & $5(8,3)$ & $17(28,3)$ \\
\hline Score MASEI & 0,166 & 0,98 & 1,33 & 0,83 & 0,31 & 0,63 & SO \\
\hline Score GUESS & 0,31 & 0,76 & 0,97 & 0,65 & 0,2 & SO & SO \\
\hline \multicolumn{8}{|l|}{ TS $(n=52)$} \\
\hline Épaisseur & $3(5,7)$ & $28(53,8)$ & $35(67,3)$ & $3(5,7)$ & $7(13,4)$ & $1(1,9)$ & $2(3,8)$ \\
\hline $\begin{array}{l}\text { Hypo- } \\
\text { échogénicité }\end{array}$ & $1(1,9)$ & $0(0)$ & $0(0)$ & $0(0)$ & $0(0)$ & $0(0)$ & $4(7,6)$ \\
\hline Positivité DP & $0(0)$ & $0(0)$ & $0(0)$ & $0(0)$ & $0(0)$ & $1(1,9)$ & $5(9,6)$ \\
\hline Calcifications & $2(3,8)$ & $1(1,9)$ & $0(0)$ & $0(0)$ & $0(0)$ & $2(3,8)$ & $2(3,8)$ \\
\hline Enthésophytes & $12(23,0)$ & $0(0)$ & $0(0)$ & $5(9,6)$ & $3(5,7)$ & $2(3,8)$ & $6(11,5)$ \\
\hline Érosions & $0(0)$ & $0(0)$ & $0(0)$ & $0(0)$ & $0(0)$ & $1(1,9)$ & $2(3,8)$ \\
\hline Score MASEI & 0,11 & 0,55 & 0,67 & 0,15 & 0,06 & 0,17 & SO \\
\hline Score GUESS & 0,30 & 0,57 & 0,67 & 0,25 & 0,21 & SO & SO \\
\hline
\end{tabular}

Les données sont présentées sous la forme nombre (pourcentage) de sites d'enthèses.

TQF : tendon du quadriceps fémoral ; IPTP : insertion proximale du tendon patellaire ; IDTP : insertion distale du tendon patellaire ; TA : tendon d'Achille ; FP : fascia plantaire ; EL :

épicondyles latéraux ; DP : Doppler Puissance ; SO : sans objet ; MASEI : Madrid Sonographic Enthesitis Index; GUESS : Glasgow Ultrasound Enthesitis Scoring System. 